\title{
Altruistic Versus Rational Behavior in a Public Good Game
}

\author{
Alexander Matros* \\ 4713 WW Posvar Hall \\ DEPARTMENT OF ECONOMICS \\ University OF PitTSBURGH \\ PitTsburgh, PA 15260 \\ E-MAIL: ALM75@PITT.EDU
}

October 3, 2008

\begin{abstract}
This paper analyses an evolutionary version of the Public Good game of Eshel, Samuelson, and Shaked (1998) in which agents can choose between imitation and best-reply decision rules. We describe conditions under which altruistic and rational (maximizing) behavior arise: these conditions are established for any number of neighbors and any total number of agents in the population. Given mistake-free play, (short-run) outcomes are identical whether agents are constrained to employ an imitation rule only; or they can choose between imitation and best-reply rules. Given the possibility of mistakes, (long-run) outcomes vary across these two scenarios. The paper suggests how to provide public goods and gives an explanation of why we observe seemingly irrational cooperation - altruistic behavior - in the rational world. (JEL C70, C72, C73)
\end{abstract}

\footnotetext{
*I would like to thank Ken Binmore, Tilman Borgers, David DeJong, John Duffy, Bard Harstad, Burkhard Hehenkamp, Steffen Huck, Sergey Izmalkov, Jens Josephson, Jack Ochs, Rafael Rob, and seminar participants at University College London, Exeter University, New Economic School, University Texas A\&M, University of Pittsburgh, and McGill University for their comments.
} 
"... individual firms behave as if they were seeking rationally to maximize their expected returns..."

Milton Friedman (1953, page 21.)

"... success (survival) accompanies relative superiority..."

Armen A. Alchian (1950, page 213.)

\section{INTRODUCTION}

The preceding characterizations of firm behavior reflect a consensus view among economists. Yet examples of seemingly contradictory behavior abound. This paper offers a potential evolutionary explanation how altruistic behavior can arise in the rational world.

It is well understood in the evolutionary literature that altruistic behavior can prevail in an evolutionary environment if all agents are imitators and their interactions are local. See Bergstrom and Stark (1993); Eshel, Samuelson, and Shaked (1998); and Bergstrom (2002).

The present paper also uses the local interaction structure: an evolutionary version of the Public Good game of Eshel, Samuelson, and Shaked (1998), but my agents select a rule (either imitation or best-reply) in every period, and then use this rule to choose a strategy. The selected rule can be the same decision rule or may vary across agents and periods. I demonstrate that altruistic behavior has hope in this world.

The Public Good game is considered for several reasons. First, it is simple: every player has just two strategies in the Public Good game. Second, one strategy strictly dominates the other and corresponds to spiteful and rational behavior. In other 
words, Armen Alchian and Milton Friedman will agree on this strategy. The other strategy is strictly dominated and corresponds to altruistic behavior.

Third, the imitation rule is hardly the only decision rule that can be used by experienced economic agents. Moreover, we cannot ignore the fact that firms use rational rules. There are many rational rules. ${ }^{1}$ But without loss of generality we can restrict our analysis only to the one of such rules - the best-reply rule, because any rational decision rule selects the dominant strategy in the Public Good game.

Finally, there is a possible conflict between imitation and best-reply decision rules: On the one hand Eshel, Samuelson, and Shaked (1998) show that the imitation rule can lead to altruistic behavior in the Public Good game, even in the presence of mutations that continually introduce the rational strategy into the model. On the other hand, Young (1998) demonstrates that the best-reply rule leads to rational behavior.

In my model, the agents select the best-performing decision rule from the previous period. In order to make the exposition simple, I assume that an agent uses the previous-period decision rule, if this rule selected the strategy with the highest payoff in her neighborhood in the previous period, and switches to the other rule otherwise.

My assumption that agents can use several decision rules is supported in experiments. The literature on experimental economics claims that there is no single rule that can describe human behavior (see Mookherjee and Sopher, 1994, 1997; Cheung and Friedman, 1997; Camerer and Ho, 1999; Huck, Normann and Oechssler, 1999; Salmon, 2001).

In reality, from time to time humans experiment with many different decision rules. (See Arthur, 1994, for an excellent discussion. He also cites psychology literature.) Arthur (1994) models agents with several predictions or hypotheses in the form of

\footnotetext{
${ }^{1}$ See, for example, Stahl (1993), Saez-Marti and Weibull (1999) and Matros (2003).
} 
functions that map previous outcomes into next-period outcomes. However, he offers only computer simulation for a particular "Bar Problem".

My main result is that altruistic behavior can arise in both the short run (mistakefree play) and in the long run (in the presence of mistakes and errors), if the neighborhoods are not too small. ${ }^{2}$ It is in sharp contrast to Eshel, Samuelson, and Shaked's (1998) claim that

This [imitation] is crucial, as altruism has no hope in a world of best responders. ${ }^{3}$

This paper shows that this claim is not precise. Moreover, if altruistic behavior survives in the short and long run, agents demonstrate altruistic behavior in groups: agents obtain high payoffs from altruistic behavior of neighbors in such groups and do not switch (imitation) decision rule and therefore do not change behavior.

I demonstrate that one of the two decision rules, the imitation rule, fully characterizes short-run outcomes. It means that even though a set of decision rules can better represent human behavior, the actual short-run prediction of the model (mistake-free play) can coincide with a simpler model where each agent has just one decision rule.

Even though the imitation rule is enough to characterize agents' short-run behavior, Theorem 2 shows that both decision rules are important in determining long-run outcomes. Some agents can occasionally switch decision rules and/or their behavior in the long run. Again, if altruistic behavior survives, it survives in groups. This result gives an idea how to provide public goods: the provision should be organized in relatively small groups.

\footnotetext{
${ }^{2}$ In the two - neighbor case only spiteful maximizing behavior remains in the population, given the possibility of mistakes.

${ }^{3}$ Eshel, Samuelson, and Shaked (1998), page 158.
} 
Theorem 2 describes long-run outcomes, if the probabilities of agents' mistake in the selection of decision rules $(\mu \rightarrow 0)$ and error in choosing a strategy $(\varepsilon \rightarrow 0)$ tend to zero. If it is easier to make a mistake in the selection of the decision rule than to make an error in choosing a strategy to play, occasionally spiteful behavior will prevail and consequently be the only possible behavior in the long run (if $\varepsilon \rightarrow 0$ faster than $\mu \rightarrow 0$ ). However, if it is easier to make an error in choosing a strategy to play than to make a mistake in the selection of the decision rule $(\mu \rightarrow 0$ faster than $\varepsilon \rightarrow 0$ ), then altruistic behavior can survive in groups, because the gain to stay in altruistic groups is higher than the gain from staying in spiteful groups. Theorem 2 describes completely long-run outcomes for different speed of convergence of $\mu$ and $\varepsilon$ to zero.

The work related to mine also includes papers on the market selection hypothesis, which claims that market forces weed out less profitable firms in favor of more profitable firms. There is a growing literature on market survival, which started with Winter (1964), followed by Winter (1971), Nelson and Winter (1982), and recently taken up by Blume and Easley (1992, 1995, 2002), Radner (1996), Vega-Redondo (1997), Dutta and Radner (1999), Dutta and Sundaram (2001). These papers check the hypothesis and demonstrate that firms' decision rules, which maximize long-run survival probabilities, are not those which maximize expected profits. This is the same kind of result as we have in Theorem 2 .

Finally, the best-reply and imitation rules are typical rules in evolutionary models. The best-reply rule usually leads to a rational outcome (see Young, 1993, 1998; Kandori, Mailath, and Rob, 1993; Ellison, 1993; Blume, 1993; Samuelson, 1997). Bergstrom and Stark (1993); Eshel, Samuelson, and Shaked (1998) show that the imitation rule can lead to altruistic behavior. Simulation evidence for imitation behavior can be found in Nowak and May $(1992,1993)$ and Nowak, Bonhoeffer, and 
May (1994).

The rest of the paper is organized as follows. Section 2 describes the model. Section 3 analyses the short-run prediction of the model. Section 4 examines the long-run prediction of the model, if the agents can make mistakes and errors. Section 5 concludes. Proofs are given in the Appendix.

\section{The Model: Public Good Game}

We will call the following game the Public Good game. There are N players. A player can either produce public goods which give one unit of utility to $2 k<N$ of her neighbors and incurs a net cost of $0<c<1$ to the player herself, or do nothing at no cost. In other words, every player has two strategies: be Altruistic with neighbors and produce public goods, or be Rational and not produce public goods. We will call these strategies $A$ and $R$ respectively. Then the payoff of an agent $i$ is $\pi_{i}=K(A, i)-c$, if agent $i$ plays strategy $A$, and $\pi_{i}=K(A, i)$, if agent $i$ plays strategy $R$, where $K(A, i) \in\{0, \ldots, 2 k\}$ is the number of $i$ 's neighbors who play strategy $A$.

The one-shot Public Good game has one strict Nash equilibrium $(R, \ldots, R)$, where all players play strategy $R$. Moreover, strategy $A$ is strictly dominated by strategy $R$. If the Public Good game is played repeatedly, the Folk Theorem can be applied and the play $(A, \ldots, A)$, where each player plays strategy $A$, can be sustained as an equilibrium in the infinitely repeated Public Good game. ${ }^{4}$

We will consider the following evolutionary version of the Public Good game. In each discrete time period, $t=1,2, \ldots$, a population of $N$ boundedly rational agents plays the Public Good game. ${ }^{5}$ We assume, as in Ellison (1993); Bergstrom and Stark

\footnotetext{
${ }^{4}$ For discussion and applications of Folk Theorem see Fudenberg and Tirole (1991).

${ }^{5}$ Boundedly rational agents follow some (simple) decision rules. There might be several reasons for that. For example, agents might lack enough abilities, or they simply do not have time to think
} 
(1993); and Eshel, Samuelson, and Shaked (1998), that the agents are located on a circle. An agent $i$ chooses a strategy $x_{i}^{t} \in\{A, R\}$ at time $t$ according to a decision rule defined below. The play at time $t$ is the vector $x^{t}=\left(x_{1}^{t}, \ldots, x_{N}^{t}\right)$.

Strategies are chosen as follows. We assume that every agent has two decision rules. They are Best Reply $(B R)$ and Imitation $(I M)$. The $B R$ rule can be described as follows. An agent believes that her neighbor agents will play the same strategies in period $t+1$ as they did in the previous period $t$. Therefore, she plays a strategy in period $t+1$ which is the best reply to her $2 k$ neighbors' strategy distribution $\left(x_{i-k}^{t}, \ldots, x_{i-1}^{t}, x_{i+1}^{t}, \ldots, x_{i+k}^{t}\right)$ from the previous period $t$. Note that since strategy $R$ is the dominant strategy, the $B R$ rule always chooses strategy $R$. The $I M$ rule works as follows. An agent plays a strategy in period $t+1$, which gave the highest payoff among her $2 k$ neighbors and her in the previous period $t$.

Now we explain how agents select decision rules. Suppose that an agent $i$ used a decision rule $D^{t} \in\{B R, I M\}$ and played a strategy $\in\{A, R\}$ in period $t$. Define the set of neighbors of player $i$ as

$$
J_{i} \equiv\{i-k, \ldots, i+k\}
$$

Agent $i$ inspects a sample $\left(x_{i-k}^{t}, \ldots, x_{i-1}^{t}, x_{i}^{t}, x_{i+1}^{t}, \ldots, x_{i+k}^{t}\right)$ of size $2 k+1$ of her $2 k$ nearest neighbors and her, taken from the previous play at time $t$ and the corresponding payoffs $\left(\pi_{i-k}^{t}, \ldots, \pi_{i-1}^{t}, \pi_{i}^{t}, \pi_{i+1}^{t}, \ldots, \pi_{i+k}^{t}\right)$ in period $t$. Denote by

$$
J_{i}(x, t) \equiv\left\{j \in J_{i} \mid x_{j}^{t}=x\right\}
$$

the set of neighbors who chose strategy $x \in\{A, R\}$ in period $t$. Then agent $i$ finds the maximum payoff in the sample:

$$
\pi_{i}^{*}\left(x^{t}\right)=\max \left\{\pi_{j}^{t} \mid j \in J_{i}\right\}
$$

about the situation. 
and the maximum payoff to her previous period strategy:

$$
\pi_{i}^{*}\left(x_{i}^{t}, t\right)= \begin{cases}\max \left\{\pi_{j}^{t} \mid j \in J_{i}(A, t)\right\}, & \text { if } x_{i}^{t}=A, \\ \max \left\{\pi_{j}^{t} \mid j \in J_{i}(R, t)\right\}, & \text { if } x_{i}^{t}=R .\end{cases}
$$

If $\pi_{i}^{*}\left(x_{i}^{t}, t\right)=\pi_{i}^{*}\left(x^{t}\right)$, the agent $i$ uses the decision rule $D^{t}$ in period $t+1$ again, otherwise she switches to the decision rule $D^{t+1} \neq D^{t}$ in period $t+1$. There is exactly one strategy in every sample which gives the highest payoff, because of our assumption $0<c<1$. An interpretation of such a rule selection is the following. If an agent's current strategy did not attain the maximum payoff in the neighborhood, whatever decision rule led to that choice does not seem to work well, so the agent switches her decision rule.

Note that the agents do not observe the decision rules of other agents, just their strategies. The assumption which rule to use in the current period is important and we will look at the following examples to see how agents can change the imitation rule into the best-reply rule and vice versa. We will call an agent whose previous period decision rule is $B R$, a maximizer, and an agent whose previous period decision rule is $I M$, an imitator. We will say that an agent behaves altruistically if her previous period strategy is $A$, and rationally if her previous period strategy is $R .{ }^{6}$ Therefore we can have rational maximizers and imitators and altruistic imitators in the model.

Example 1. Suppose that $k=2$ and $N>5$. Suppose that an imitator observes the following sample

$$
\left(x_{i-2}^{t}, x_{i-1}^{t}, x_{i}^{t}, x_{i+1}^{t}, x_{i+2}^{t}\right)=(A, R, \underline{A\{I M\}}, A, A)
$$

and the corresponding payoffs

$$
\left(\pi_{i-2}^{t}, \pi_{i-1}^{t}, \pi_{i}^{t}, \pi_{i+1}^{t}, \pi_{i+2}^{t}\right)=(3-c, 4,3-c, 3-c, 4-c)
$$

\footnotetext{
${ }^{6}$ Note that in the model both spiteful behavior and optimizing behavior correspond to the same strategy $S$. We will call such behavior spiteful.
} 
where $\underline{A}$ is her own strategy choice. Only one agent plays strategy $R$ in the sample and the other four agents play strategy $A$. It is obvious that

$$
\pi_{i}^{*}\left(x^{t}\right)=\pi^{*}(R)=4>4-c=\pi^{*}(A)
$$

and the imitator will become the maximizer in the next period.

Suppose now that a maximizer observes the following sample

$$
\left(x_{i-2}^{t}, x_{i-1}^{t}, x_{i}^{t}, x_{i+1}^{t}, x_{i+2}^{t}\right)=(A, A, \underline{R\{B R\}}, R, R)
$$

and the corresponding payoffs

$$
\left(\pi_{i-2}^{t}, \pi_{i-1}^{t}, \pi_{i}^{t}, \pi_{i+1}^{t}, \pi_{i+2}^{t}\right)=(3-c, 2-c, 2,1,0)
$$

where two of her neighbors play strategy $R$, the other two play strategy $R$ and $\underline{R}$ is her own strategy choice. Since

$$
\pi_{i}^{*}\left(x^{t}\right)=\pi^{*}(A)=3-c>2=\pi^{*}(R),
$$

the maximizer will become the imitator in the next period.

Assume that the sampling process begins in the period $t=1$ from some arbitrary initial play $x^{0}$ and some arbitrary initial decision rule distribution $d^{0}$. Then we obtain a finite Markov chain on the finite state space $(\{A, R\})^{N} \times(\{B R, I M\})^{N}$ of states of the length $2 N$ drawn from the strategy space $\{A, R\}$ and the decision rule space $\{B R, I M\}$ with an arbitrary initial play $x^{0}$ and some arbitrary initial decision rule distribution $d^{0}$. Given a play $x^{t}$ and a decision rule distribution $d^{t}$ at time $t$, the process moves to a state of the form $\left\{x^{t+1} ; d^{t+1}\right\}$ in the next period. Such a state is called a successor of $\left\{x^{t} ; d^{t}\right\}$. We will call this process unperturbed adjusted dynamic with population size $N$ and $2 k$ neighbors, $A D^{N, k, 0,0}$.

Example 2. Suppose that $k=1, N=4, x^{0}=(A, A, R, R)$ and $d^{0}=(I M, I M, B R, B R)$. 
In period 1 , each agent $i$ inspects a sample $\left(x_{i-1}^{0}, x_{i}^{0}, x_{i+1}^{0}\right)$ of size 3 of her 2 nearest neighbors and her, taken from the previous play in period 0. Agents 3 and 4 (1 and 2) use the $B R(I M)$ rule to choose a strategy at the beginning of period 0. Agents 3 and 4 will use the $B R$ rule again in period 1, because these agents observe that strategy $R$ gives a payoff of 1 and strategy $A$ gives a payoff of $1-c$ (there is exactly one spiteful maximizer neighbor and one altruistic imitator neighbor for every agent). Therefore, strategy $R$ gives the highest payoff in any sample:

$$
\pi_{i}^{*}\left(x^{0}\right)=\pi^{*}(R)=1>1-c=\pi^{*}(A) \text {, for any } i .
$$

It means that agents 1 and 2 will switch to the best-reply rule, $B R$, because they also observe that strategy $R$ gives the highest payoff in their samples. Therefore $d^{1}=(B R, B R, B R, B R)$. Hence, all agents will use the $B R$ rule in period 1 and play the dominant strategy $R, x^{1}=(R, R, R, R)$. As a result the unperturbed adjusted dynamic process moves to the state $\left\{x^{1} ; d^{1}\right\}$ in period 1 .

The unperturbed adjusted dynamic process describes short-run behavior in the model when there are no mistakes and errors in the agents' behavior. Short-run predictions are useful, because the predicted outcome(s) arise very fast, due to the local interaction structure of the model, stay long (until a mistake or an error is made), and depend on the initial state.

Let us introduce some noise into the model. To model the situation in which a rule can be chosen by a mistake, we suppose that the agents use the selected rule with probability $1-\mu$ and use the other rule at random with probability $\mu \geq 0$. Moreover, suppose that agents use a decision rule to choose a strategy with probability $1-\varepsilon$ and make an error and choose a strategy at random with probability $\varepsilon \geq 0$. The resulting perturbed adjusted dynamic process $A D^{N, k, \mu, \varepsilon}$ is an ergodic Markov process on the finite state space $(\{A, R\})^{N} \times(\{B R, I M\})^{N}$. Thus, in the long run, the initial 
state is irrelevant.

\section{Short Run: Recurrent Classes}

In the rest of the paper, we will make use of the following definitions. A recurrent class of the process $A D^{N, k, 0,0}$ is a set of states where there is a probability zero of moving from any state in the set to any state outside and there is a positive probability of moving from any state in the set to any other state in the set. We will call a state $h$ absorbing if it constitutes a singleton recurrent class. In this section we analyze the situation when all agents always use the selected rule, $\mu=0$, and do not make errors, $\varepsilon=0$.

3.1. $\mu=0$ and $\varepsilon=0$. Note that if every agent uses the imitation rule and plays strategy $A$, then every agent obtains the same payoff and therefore selects the imitation rule again in the next period. Moreover, as everyone plays the same strategy, the imitator chooses strategy $A$ in the next period as well. It means that a state of the form:

$$
\{(A, \ldots, A) ;(I M, \ldots, I M)\}=\{A ; I M\}
$$

is absorbing.

Suppose that all agents played strategy $R$ in the previous period. Either an agent used the $I M$ or the $B R$ rule, she observes only strategy $R$ in her sample. It means that she will select her previous period decision rule in the next period too. Both the imitator and the maximizer will again choose strategy $R$ in the next period. Hence, states in the form:

$$
\left\{(R, \ldots, R) ;\left(d_{1}, \ldots, d_{N}\right)\right\}
$$

are absorbing, where $d_{j}$ can be either $B R$ or $I M, j \in\{1, \ldots, N\}$. Let a set $\{(R ; \cdot)\}$ denote all $2^{N}$ states of the form (1).

The following examples show that there are other recurrent classes. 
Example 3. Suppose that $N=7$ and $k=2$.

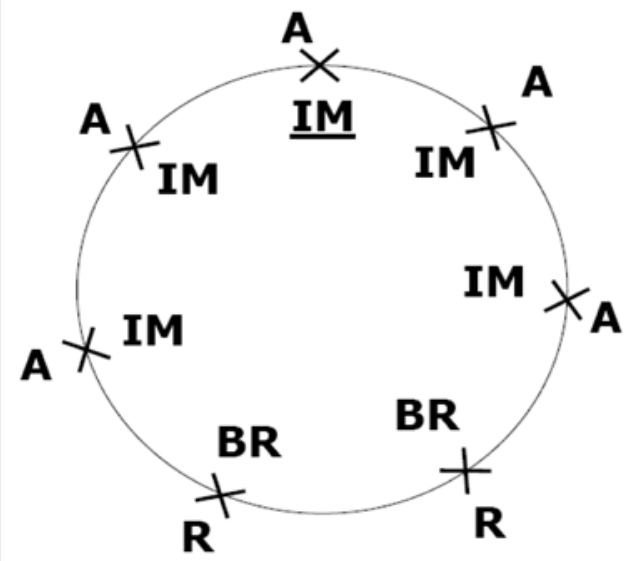

Figure 1.
$\mathrm{N}=7, \mathrm{k}=\mathbf{2}$

Best imitator, she is marked $\underline{\mathbf{I M}}$, plays strategy $\mathbf{A}$ and gets 4-c.

Both maximizers play strategy $\mathbf{R}$ and receive 3 .

Every agent uses her previous period decision rule in the next period again.

There are five imitators who play strategy $A$ and two maximizers who play strategy $R$. It is evident that every agent finds that her strategy performs best in her sample. For example, every imitator has the best imitator, marked $\underline{I M}$, in the sample. The best imitator receives $4-c$ which is the highest payoff among all agents. It means that for each imitator

$$
\pi^{*}(A)=4-c>3=\pi^{*}(R)
$$

and she selects the imitation rule in the next period.

Both maximizers do not have the best imitator in their neighborhoods which means that their payoffs are the highest in their samples:

$$
\pi^{*}(R)=3>3-c=\pi^{*}(A) .
$$

Therefore, both maximizers select the best-reply rule in the next period. It means that every agent selects her previous period decision rule again. Hence, all agents choose the previous period strategies again. Figure 1 gives an example of this recurrent class. 
The following example shows that a recurrent class can contain several states.

Example 4. There are eight imitators playing strategy $A$ and one maximizer playing strategy $R$, in the first circle in Figure 2.

\section{$\mathbf{N}=\mathbf{9}, \mathbf{k}=\mathbf{2}$}

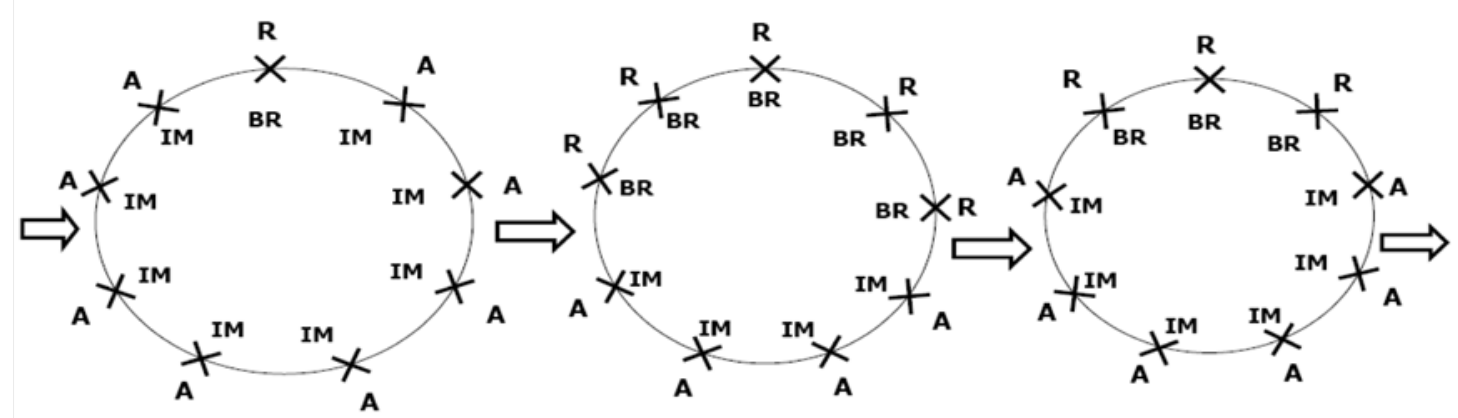

Figure 2 .

The maximizer obtains payoff 4 which is the highest payoff among all agents. Therefore, all her four neighbors find that

$$
\pi^{*}(R)=4>4-c=\pi^{*}(A)
$$

These four agents will change their decision rule from the imitation rule to the bestreply rule in the next period. All maximizers choose strategy $R$ in the next period, as it is shown in the second circle in Figure 2. There are five maximizers and four imitators in the second circle. The imitators find that

$$
\pi^{*}(A)=3-c>2=\pi^{*}(R)
$$

and select the imitation rule in next period which leads to the continuation of playing strategy $A$. Two maximizers on the boundary between the maximizers and the imitators are in the situation from example 1. They observe that two neighbors play 
strategy $A$ and the other two play strategy $R$. The highest payoff to strategy $A$ is equal to $3-c$ which is greater than the highest payoff to strategy $R$ in the sample, or

$$
\pi^{*}(A)=3-c>2=\pi^{*}(R)
$$

Therefore, these two maximizers change their decision rule from the best-reply to the imitation rule in the next period. We observe this in the third circle in Figure 2.

There are three maximizers and six imitators in the third circle. Once again, all imitators select the imitation rule and play strategy $A$ in the next period. Two maximizers on the boundary between the maximizers and the imitators are in the situation from example 1. Therefore, these two maximizers change their decision rule from the best-reply to the imitation rule in the next period. It explains how the process moves from the third to the first circle in Figure 2. The cycle repeats again. Hence, Figure 2 gives an example of a recurrent class which contains three states.

Taking these examples into consideration, we can provide a complete description of recurrent classes, if $k \geq 2$. The following are recurrent classes:

- The state where all agents are imitators playing strategy P.

- The states where all agents play strategy $R$. The set $\{(R ; \cdot)\}$ contains all the states of this kind.

- The state where all agents are imitators playing strategy $A$ except for the two adjacent imitators or maximizers playing strategy $R$ :

$$
\ldots A, \ldots, A, R, R, A, \ldots A, \ldots
$$

- The set of three states, consisting of:

$$
\ldots A, \ldots A, R, A, \ldots A, \ldots
$$




$$
\begin{aligned}
& \ldots A, \ldots A, R, \ldots, R, A, \ldots A, \ldots \\
& \ldots A, \ldots, A, R, R, R, A, \ldots A, \ldots,
\end{aligned}
$$

where there is one agent playing strategy $R$ in period $t ; 2 k+1$ agents playing strategy $R$ in period $t+1$; three agents playing strategy $R$ in period $t+2$. This cycle is repeated after that.

These examples and combinations based on them include all the possible recurrent classes. Both strategies must appear in clusters in all recurrent classes.

Theorem 1. Suppose that $k \geq 2$ and $N \geq k+4$. The recurrent classes of the unperturbed process $A D^{N, k, 0,0}$ are

(i) the states where all agents play strategy $R$;

(ii) the state where all agents are imitators playing strategy $A$;

(iii) the state where a cluster of imitators consisting of $k+2$ agents or more playing strategy $A$ is separated by a cluster of imitators or maximizers consisting of two agents playing strategy $R$;

(iv) the sets of three states: in each of these states a cluster of imitators consisting of $k+2$ agents or more playing strategy $A$ is separated by a cluster of imitators or maximizers consisting of $2 k+1,3$ or 1 agents playing strategy $R$. If the cluster consists of $2 k+1$ (3 or 1 ) agents playing strategy $R$ in one of the states, the state moves to the state where the cluster consists of $3(1$ or $2 k+1)$ agents playing strategy $R$ in the next period;

(v) combinations of (iii) and (iv).

Proof. See the Appendix.

Theorem 1 indicates that there are many recurrent classes in general. In all but one of these recurrent classes, the majority of the population shows altruistic 
behavior. Moreover, Theorem 1 not only describes short-run behavior of the agents, but also emphasizes that such behavior can be described by the imitation decision rule alone. This result might explain why we could consider one decision rule in evolutionary models, even though people can use several decision rules. However, Theorem 1 proves this result only for the Public Good game. ${ }^{7}$ Since the imitation rule characterizes completely short-run outcomes for any size of the population, $N$, and the neighborhoods, $k$, Theorem 1 generalizes the short-run results from Eshel, Samuelson and Shaked (1998). ${ }^{8}$ It indicates all recurrent classes in general (even if the agents use only the imitation decision rule). To see what lies behind the result, it is helpful to remember that the imitation rule determines short-run outcomes. Even though agents use two decision rules, once a cluster of $k+2$ or more altruistic imitators is formed, every agent in this cluster finds that altruistic behavior leads to the highest payoff in the neighborhood and therefore does not change her decision rule which, in turn, selects altruistic behavior. However, a cluster of spiteful maximizers can never contain more than $2 k+1$ agents (the only exception is when all agents are spiteful maximizers) in the recurrent class. If there are more than $2 k+1$ agents in such a cluster, then the two agents at its edges will each have $k$ altruistic imitators on one side and $k$ spiteful maximizers on the other. In this situation the highest payoff in the neighborhood will correspond to the altruistic behavior which means that these two agents change their decision rule and also become altruistic imitators.

3.2. Fixed $\mu, 0<\mu<1$. This subsection clarifies the main difference between the approach of this paper and the approach of other evolutionary papers in the field. Typically authors model multiple decision rules in the following way: there are $N$

\footnotetext{
${ }^{7}$ An open question is whether one decision rule can always describe the short-run behavior of agents who use several decision rules.

${ }^{8}$ Our case $k=2$ corresponds to their main case.
} 
populations corresponding to $N$ player positions in the game. Agents are chosen at random from populations (one agent per population) to play the corresponding player positions in the game in every period. Each population consists of agents of different types: agents of the same type always use the same decision rule (see Saez-Marti and Weibull, 1999; Matros, 2003).

If $0<\mu<1$ and fixed, the agents can use both the best-reply and the imitation rules with a positive probability in every period. Another interpretation of this situation is that there is a population of agents for every player position with at least $\mu$ share of agents using the best-reply and the imitation rules.

If agents can use both decision rules with a positive probability in every period, only spiteful behavior will survive in the short run. The intuition is simple: if occasionally all agents use the best-reply rule in one period (this can happen with positive probability), only strategy $R$ will be present thereafter. The following proposition is a corollary of Matros (Theorem 1, 2004).

Proposition 1. If $\mu$ is fixed and such that $0<\mu<1$, only states from the set $\{(R ; \cdot)\}$ are absorbing states of the unperturbed process $A D^{N, k, \mu, 0}$.

This finishes the description of short-run outcomes.

\section{Long Run: Selection among Recurrent Classes}

We now ask if altruistic behavior survives in the presence of agents' mistakes or/and errors. To answer this question, long-run behavior of the adjusted dynamic process $A D^{N, k, \mu, \varepsilon}$ will be analyzed in this section. First, we analyze the situation where $\mu$ is positive and fixed (the agents can always use both decision rules) and the probabilities of errors tend to zero, $\varepsilon \rightarrow 0$. Then, we consider the situation where both mistakes and errors tend to zero, $\mu \rightarrow 0, \varepsilon \rightarrow 0$. 
4.1. Fixed $\mu, 0<\mu<1$. From Proposition 1 and Theorem 3 in the Appendix, we conclude that all agents must play strategy $R$ in the long run, because all agents play strategy $R$ in every absorbing state. This result is consistent with Matros (Theorem $2,2004)$. In other words, only spiteful behavior will be present in the population when each decision rule can be chosen in every period.

Proposition 2. If $\mu$ is fixed and such that $0<\mu<1$, the limiting distribution of the adjusted dynamic process $A D^{N, k, \mu, \varepsilon}$ puts a positive probability only on states from the set $\{(R ; \cdot)\}$, where all agents play strategy $R$.

Proposition 2 not only describes long-run behavior of the agents, but also emphasizes that such behavior can be described by just one decision rule, if the agents can use any decision rule in every period. This result holds in general, see Matros (2004). In the next subsection we find the long-run outcomes, if both mistakes and errors tend to zero, $\mu \rightarrow 0, \varepsilon \rightarrow 0$.

4.2. $\mu \rightarrow 0, \varepsilon \rightarrow 0$. Now we consider how agents' behavior changes in the presence of mistakes and errors. For this purpose we study the agents' long-run behavior when the probabilities of mistakes and errors tend to zero, $\mu \rightarrow 0, \varepsilon \rightarrow 0$. From Theorem 1 it follows that there are five "types" of the recurrent classes, if $k \geq 2$. The idea behind this section is based on the following observation. When mistake and error rates are small, the process spends almost all time in the recurrent classes. There are two possibilities to move from one recurrent class to another. Agents have to make mistakes in their choices of the decision rules (this can happen with probability $\mu$ ), or make errors in choosing a strategy (this can happen with probability $\varepsilon$ ) in order to move from a recurrent class of one type to a recurrent class of another type. We are interested in changes of the agents' behavior or the movements between the recurrent classes of different types. 
Even though Theorem 1 identifies the same recurrent classes in the case of two decision rules and the imitation rule only, we have to take into account the bestreply rule when we are looking for the long-run prediction of the model. The reason is simple: a maximizer always demonstrates spiteful behavior. It means that if it is easier to make a mistake in the selection of the decision rule than to make an error in choosing a strategy to play, occasionally spiteful behavior will prevail and consequently be the only possible behavior in the long run (if $\varepsilon \rightarrow 0$ faster than $\mu \rightarrow 0$ ). It means that only spiteful behavior will survive in the long run, because such behavior is unaffected by the switching of the decision rules. However, if it is easier to make an error in choosing a strategy to play than to make a mistake in the selection of the decision rule $(\mu \rightarrow 0$ faster than $\varepsilon \rightarrow 0)$, then altruistic behavior can survive in groups, because the gain to stay in altruistic groups is higher than the gain from staying in spiteful groups.

We are looking for the limiting distribution as the mistake, $\mu$, and error, $\varepsilon$, probabilities tend to zero. We will assume that $\mu=\mu(\varepsilon), \mu(0)=0$ and analyze the limiting distribution when $\varepsilon \rightarrow 0$. The following main theorem describes completely long-run outcomes for different speed of convergence of $\mu$ and $\varepsilon$ to zero.

Theorem 2. Suppose that $k \geq 2$.

1. If $\lim _{\varepsilon \rightarrow 0} \frac{\mu(\varepsilon)}{\varepsilon}<\infty$ and $N>4(k+1)(k+2)$, the limiting distribution of the adjusted dynamic process $A D^{N, k, \mu, \varepsilon}$ puts a positive probability on all recurrent classes except for the absorbing states where all agents play strategy $R$.

2. If $\lim _{\varepsilon \rightarrow 0} \frac{\mu(\varepsilon)}{\varepsilon}=\infty$, or $N<4(k+1)(k+2)$, the limiting distribution of the adjusted dynamic process $A D^{N, k, \mu, \varepsilon}$ contains only the absorbing states where all agents play strategy $R$.

3. If $\lim _{\varepsilon \rightarrow 0} \frac{\mu(\varepsilon)}{\varepsilon}<\infty$ and $N=4(k+1)(k+2)$, the limiting distribution of the adjusted dynamic process $A D^{N, k, \mu, \varepsilon}$ puts a positive probability on all recurrent 
classes.

Proof. See the Appendix.

The proof of the theorem shows that it is much easier to introduce altruistic behavior in the spiteful world than vice verse, as long as the population size is sufficiently large and it is easier to make an error in choosing a strategy to play than to make a mistake in the selection of the decision rule. The intuition for this result is as follows. It takes only one mistake or error to introduce spiteful maximizer (imitator) into a world of altruistic imitators. However, the resulting group of spiteful agents cannot become more than $2 k+1$. To get additional spiteful agents, additional mistakes or/and errors are required. These mistakes or/and errors can lead to states where there are many groups of spiteful agents. If two such groups join together after a mistake or error, then the new group of spiteful agents will contain more than $2 k+1$ agents and this group will shrink in the next period in the presence of an altruistic group of agents in the population. The only chance to create a spiteful world arises if there are mistakes, or errors in every altruistic group of agents. The number of such mistakes or errors is large for large $N$, because between every two spiteful groups of agents there must be a group of altruistic agents.

Theorem 2 describes for what parameters $N$ and $k$ the long-run prediction of the model contains altruistic behavior of agents. If $\mu=\varepsilon$, or mistakes and errors are equally likely, the main condition of the Theorem becomes a comparison between the population size, $N$, and $4(k+1)(k+2)$. If we fix $k$ such that $2 k+1=N$, then the unique prediction of the model is spiteful behavior of all agents. Now, if we keep fixed $k$ and start to increase the number of agents on the circle, $N$, spiteful behavior - all agents play strategy $S$ - will be the only long-run prediction until $N=4(k+1)(k+2)$. If we increase the number of agents, $N$, further, altruistic behavior appears. Note 
that altruistic behavior will appear if the number of agents on the circle is much greater that the number of neighbors. Similar effects arise if the number of agents on the circle, $N$, is fixed and we start to decrease the number of neighbors, $k$, down to 2 .

One might expect that trembles in behavior are higher order than in selection between behavior rules - people change behavior rules more consciously and deliberately than their actions. That would correspond to the case where $\mu$ goes to zero faster than $\varepsilon(\mu \rightarrow 0$ faster than $\varepsilon \rightarrow 0)$, the argument similar to the case $\mu=\varepsilon$ works and a comparison between the population size, $N$, and $4(k+1)(k+2)$ determines the agents' long-run behavior. This has an important bearing on the survival of "simple" models of behavior in the face of competition from more sophisticated ones: Theorem 2 describes long-run outcomes, as if all agents would use only the imitation decision rule. The comparison between the population size, $N$, and $4(k+1)(k+2)$ defines the agents' long-run behavior. Theorem 2 generalizes the results of Eshel, Samuelson and Shaked's (1998) model for any population size, $N$, and any size of the neighborhoods, $k$.

Theorem 2 also suggests how to provide public goods: the population should be divided in (relatively) small neighborhoods in which everybody can observe the nearest neighbors' contributions. This can lead to altruistic behavior and to the provision of the public goods.

\section{CONCLUSION}

This paper analyzes an evolutionary version of the Public Good game in which agents can use two decision rules. This framework is modeled as a Markov chain on a finite state space. It is shown (Theorem 1 ) that if $k \geq 2$, short-run behavior of the system can have five types: (i) all agents are altruistic, (ii) all agents are rational, (iii) there are two types of agents so that between two clusters of altruistic agents there is a 
cluster of two rational agents, (iv) there are two types of agents so that between two clusters of altruistic agents there is a cycle cluster when the number of rational agents in the cluster varies from one to $2 k+1$, then to three and then back to one, or $(\mathrm{v})$ different combinations of (iii) and (iv).

This result is consistent with the results in Bergstrom and Stark (1993) and Eshel, Samuelson, and Shaked (1998), where agents use only the imitation rule and have four, $k=2$, or six, $k=3$, neighbors. ${ }^{9}$ One of the contributions of this paper is to show that this result is robust to the introduction of the best-reply rule into the system. Moreover, Theorem 1 is a generalization of the previous results for any population size, $N$, and any size of the neighborhoods, $k$.

Theorem 1 also reveals that it is enough to have just one decision rule in order to obtain the same short-run outcomes as we obtain in the case of two decision rules. It means that we can use simple models with just one decision rule to analyze human behavior: the short-run prediction is the same in more complicated models. There are open and interesting questions for future research. Is it always the case that for any set of decision rules, $\Psi$, and any game $\Gamma$ there always exists a unique decision rule $\psi\left(\psi^{\prime}\right)$ such that the short- (and long-) run predictions if the agents use the set of decision rules $\Psi$, or just one decision rule $\psi\left(\psi^{\prime}\right)$ is the same for playing game $\Gamma$ ? ${ }^{10}$

I demonstrate that, even with the possibility of using the best-reply rule, altruistic behavior can be sustained not only in the short run, but also in the long run. At the same time, Theorem 2 shows the limit of Eshel, Samuelson, and Shaked (1998) results

\footnotetext{
${ }^{9}$ Bergstrom and Stark (1993) and Eshel, Samuelson, and Shaked (1998) compare the average payoffs instead of the highest payoffs. In their setting, the number of the neighbors can be two, or four. These cases correspond to the cases $k=2$ and $k=3$ in my model.

${ }^{10}$ This paper describes the short- and long- run outcomes in the Public Good Game. It is the first step in our understand of the short- and long- run predictions in general games where agents can use several decision rules.
} 
for an arbitrary number of neighbors. They fix the number of the neighbors, four or six, and find the total number of agents, $N$, so that altruistic behavior survives in the long run. Theorem 2 describes long-run outcomes for any number of neighbors, $k$, and for any total number of agents, $N$.

My model also suggests how to provide public goods: the population should be divided in (relatively) small neighborhoods where everybody can observe the nearest neighbors' contributions. This leads to altruistic behavior and to the provision of the public goods.

\section{APPENDIX}

Proof of Theorem 1: It is obvious that the states where all agents are playing strategy $R$ or all agents are imitators playing strategy $A$ are absorbing.

To find the remaining recurrent classes consider what happens to a cluster of imitators playing strategy $A$. Note that any cluster of the imitators consisting of $1,2, \ldots, k+1$ agents will immediately disappear. So, imitators can play strategy $A$ in groups of the length $k+2$ or more. Consider what happens to a cluster of agents playing strategy $R$. Any cluster consisting of three or more agents will shrink in the next period. ${ }^{11}$ It will be shrinking until the cluster of strategy $R$ becomes of two or one. The cluster of two agents playing strategy $R$ will not change. However, if there is only one agent playing strategy $R$ among her $2 k$ neighbors playing strategy $A$, the whole neighborhood - all $2 k+1$ agents - will become maximizers and will play strategy $R$ in the next period. Then this cluster of maximizers consisting of $2 k+1$ agents playing strategy $R$ shrinks to the cluster of maximizers consisting of three,

\footnotetext{
${ }^{11}$ It can stay the same size one more period if some agents use the imitation rule to select strategy $S$ in the current period. These imitators will switch their decision rule to the best-reply and will play strategy $S$ again in the next period. Imitators playing strategy $P$ in the current period will use the same rule in the next period and play strategy $P$.
} 
then one agent playing strategy $R$. This cycle will be repeated again.

We now turn our attention to the perturbed adjusted dynamic process. In particular, we are interested in the limiting distribution of this process as the mistake, $\mu$, and error, $\varepsilon$, probabilities tend to zero. We assume that $\mu=\mu(\varepsilon), \mu(0)=0$ and analyze the limiting distribution when $\varepsilon \rightarrow 0$.

By arguments similar to those in Young (1993), the perturbed adjusted dynamic process $A D^{N, k, \mu(\varepsilon), \varepsilon}$ is a regular perturbation of $A D^{N, k, 0,0}$, and hence it has a unique stationary distribution $\rho(\varepsilon)$. Moreover, by Theorem 4 in Young $(1993), \lim _{\varepsilon \rightarrow 0} \rho(\varepsilon)=$ $\rho(0)$ exists, and $\rho(0)$ is a stationary distribution of $A D^{N, k, 0,0}$.

Definition 1. (Young, 1993) A state $h$ is stochastically stable if $\lim _{\varepsilon \rightarrow 0} \rho_{h}(\varepsilon)>0$.

Let the process $A D^{N, k, \mu(\varepsilon), \varepsilon}$ have recurrent classes $E_{1}, \ldots, E_{M}$. For each pair of distinct recurrent classes, a $p q$-path is a sequence of states $\zeta=\left(h_{p}, \ldots, h_{q}\right)$ beginning in $E_{p}$ and ending in $E_{q}$. The resistance of this path is the sum of the resistances on the edges composing it. Let $r_{p q}$ be the least resistance over all $p q$-paths. Construct a complete directed graph with $M$ vertices, one for each recurrent class. The weight on the directed edge $E_{p} \rightarrow E_{q}$ is $r_{p q}$. A tree rooted at $E_{l}$ is a set of $M-1$ directed edges so that from every vertex different from $E_{l}$ there is the only possible directed path in the tree to $E_{l}$. The resistance of such a rooted tree $\Psi\left(E_{l}\right)$ is the sum of resistances $r_{p q}$ on its $M-1$ edges. The stochastic potential of a recurrent class $E_{l}$ is the minimum resistance over all trees rooted at $E_{l}$. The following theorem is analogous to results of Freidlin and Wentzell (1984) on Wiener processes. Foster and Young (1990) introduced the theorem to economics for continuous state spaces. Young $(1993,1998)$ contains a discrete version of the theorem.

Theorem 3. (Young, 1998) The stochastically stable states are precisely the states contained in the recurrent classes of $A D^{N, k, 0,0}$, having minimum stochastic potential. 
Proof of Theorem 2: From Theorem 1 follows that any recurrent class can contain $N, N-2, N-3, N-4, \ldots, k+3, k+2$, or 0 imitators playing strategy $A$. Note that it is enough to make just one mistake, $\mu(\varepsilon)$, or one error, $\varepsilon$, for moving from the recurrent class (iii) to the recurrent class (iv) from Theorem 1 and vice versa. It means that these recurrent classes have the same stochastic potential. It also means that the recurrent class $(\mathrm{v})$ will have the same stochastic potential as recurrent classes (iii) and (iv).

One error, $\varepsilon$, is enough for moving from the absorbing state $\{A ; I M\}$ (recurrent class (ii)) to an absorbing state where $N-1$ imitators are playing strategy $A$ and vice versa (recurrent class (iv)). At the same time, one mistake, $\mu(\varepsilon)$, is enough for moving from the absorbing state $\{A ; I M\}$ to an absorbing state where $N-1$ imitators are playing strategy $A$.

$(k+2)$ errors are required for moving from the absorbing state where all imitators are playing strategy $R$ (from recurrent class (i)) to the recurrent class where only 2 agents are play strategy $R$ for $N$ even (to recurrent class (iii)), or to sets of blinkers where $1,2 k+1$, or 3 agents are playing strategy $R$ (to recurrent class (iv)). These $(k+2)$ errors must create a cluster of imitators consisting of $k+2$ agents playing strategy $A$. Note that all absorbing states in the set $\{(R ; \cdot)\}$ (recurrent class (i)) have the same stochastic potential, because one mistake is enough for moving from the absorbing states $\{R ; B R\}$ to $\{(R, \ldots, R) ;(I M, B R, \ldots, B R)\}$, from $\{(R, \ldots, R) ;(I M, I M, B R, \ldots, B R)\}$ to $\{(R, \ldots, R) ;(I M, B R, \ldots, B R)\}, \ldots$, from 
$\{R ; I M\}$, to $\{(R, \ldots, R) ;(I M, \ldots, I M, B R)\}$ and vice versa.

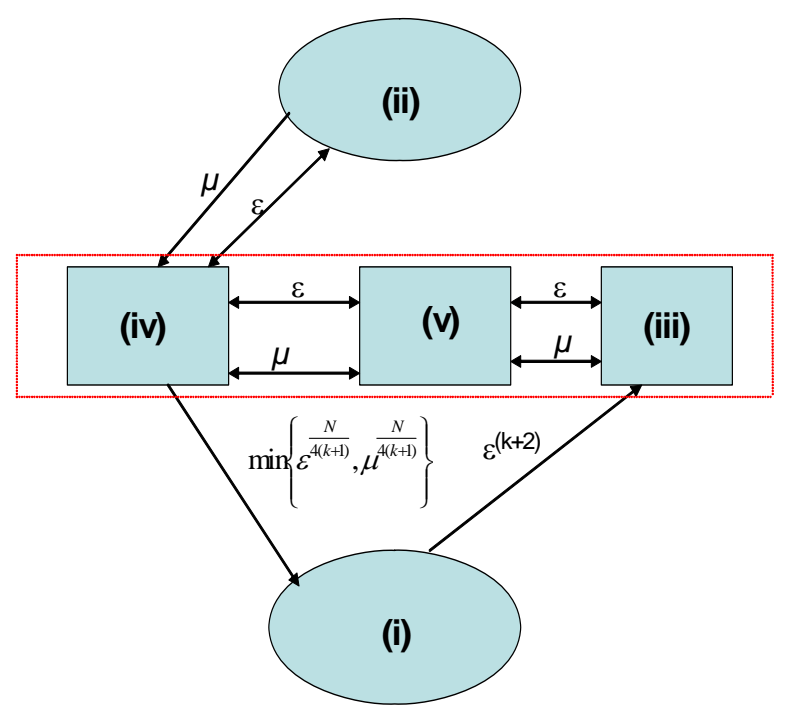

Figure 3: Recurrent Classes

What is the smallest number of mistakes and/or errors which is necessary to make for moving from the recurrent class with at least $(k+2)$ imitators playing strategy $A$ to the recurrent class where all agents are playing strategy $R$ ? Theorem 1 shows that the cluster of imitators playing strategy $A$ is at least of the length $(k+2)$ and the cluster of strategy $R$ is at most of the length $(2 k+1)$ in any recurrent class. There must be at least one mistake or one error per cluster of strategy $A$ for moving to the absorbing state where all agents are playing strategy $R$. After such a mistake or error every cluster must consist of at most $(k+1)$ imitators playing strategy $A$ in order to disappear in the next period. It is possible for a cluster of the maximal length of $(2 k+3)$. That cluster must be between two clusters of agents playing strategy $R$ and each of them consists of at most $(2 k+1)$ agents. Hence, at least

$$
\frac{N}{(2 k+3)+(2 k+1)} \min \{\varepsilon, \mu(\varepsilon)\}=\frac{N}{4(k+1)} \min \{\varepsilon, \mu(\varepsilon)\}
$$


mistakes and/or errors are necessary to move from the recurrent class (iii) or the recurrent class (iv) into an absorbing state where all agents play strategy $R$, recurrent class (i). Figure 3 summarizes all the statements above. The statement of the theorem follows immediately.

\section{REFERENCES}

[1] Alchian, Armen A. "Uncertainty, Evolution and Economic Theory." Journal of Political Economy, June 1950, 58(3), 211-21.

[2] Apesteguia, Jose; Huck, Steffen and Oechssler Jorg. "Imitation - Theory and Experimental Evidence.” Discussion Paper, 2003.

[3] Arthur, W. Brian. "Inductive Reasoning and Bounded Rationality." American Economic Review, May 1994 (Papers and Proceedings), 84(2), 406-11.

[4] Bergstrom, Theodore C. and Stark, Oded. "How Altruism Can Prevail in an Evolutionary Environment." American Economic Review, May 1993 (Papers and Proceedings), 83(2), 149-55.

[5] Bergstrom, Theodore C. "Evolution of Social Behavior: Individual and Group Selection." Journal of Economic Perspective, Spring 2002, 16(2), 67-88.

[6] Blume, Lawrence E. "The Statistical Mechanics of Strategic Interaction." Games and Economic Behavior, July 1993, 5(3), 387-424.

[7] Blume, Lawrence E. and Easley David. "Evolution and Market Behavior." Journal of Economic Theory, 1992, 58, 9-40.

[8] ____-. "Evolution and learning in competitive markets," in Kirman A. and M. Salmon (eds.), Learning and Rationality in Economics, 1995, Blackwell (Oxford). 
[9] _-_-_. "Optimality and Natural Selection in Markets." Journal of Economic Theory, November 2002, 107(1), 95-135.

[10] Camerer, Colin. and Ho Teck-Hua. "Experience - Weighted Attraction Learning in Normal Form Games." Econometrica, July 1999, 67(4), 827-74.

[11] Cheung, Yin-Wong. and Friedman Daniel. "Individual Learning in Normal Form Games: Some Laboratory Results." Games and Economic Behavior, April 1997, 19(1), 46-76.

[12] Dutta, Prajit. and Radner Roy. "Profit Maximization and the Market Selection Hypothesis." Review of Economic Studies, October 1999, 66(4), 769-98.

[13] Dutta, Prajit. and Sundaram Rangarajan. "Survival and the Art of Profit Maximization," Review of Economic Design, December 2001, 6(3-4), 429-46.

[14] Ellison, Glenn. "Learning, Local Interaction, and Coordination." Econometrica, September 1993, 61(5), 1047-72.

[15] Enke, Stephen. "On Maximizing Profits: A Distinction Between Chamberlin and Robinson." American Economic Review, September 1951, 41(4), 566-78.

[16] Eshel, Ilan; Samuelson, Larry and Shaked Avner. "Altruists, Egoists, and Hooligans in a Local Interaction Model." American Economic Review, March 1998, 88(1), 157-79.

[17] Foster, Dean. and Young, H. Peyton. "Stochastic Evolutionary Game Dynamics." Theoretical Population Biology, 1990, 38, 219-32.

[18] Freidlin, M. I. and Wentzell A. D. Random perturbations of dynamical systems. New York: Springer-Verlag, 1984. 
[19] Friedman, Milton. Essays in Positive Economics. The University of Chicago Press, 1953.

[20] Fudenberg, Drew and Tirole Jean. Game Theory. The MIT Press, Cambridge, Massachusetts, 1991.

[21] Huck, Steffen; Normann, Hans-Theo and Oechssler Jorg. "Learning in Cournout Oligopoly - An experiment." Economic Journal, March 1999, 109(454), 80-95.

[22] Kandori, Michihiro; Mailath, George J. and Rob, Rafael. "Learning, Mutation, and Long Run Equilibria in Games." Econometrica, January 1993, 61(1), 29-56.

[23] Matros, Alexander. "Clever Agents in Adaptive Learning." Journal of Economic Theory, July 2003, 111(1), 110-24.

[24] ___-_. "Simple Rules and Evolutionary Selection," mimeo, 2004.

[25] Mookherjee, Dilip. and Sopher Barry. "Learning Behavior in an Experimental Matching Pennies Game." Games and Economic Behavior, July 1994, 7(1), 6291.

[26] _____._. "Learning and Decision Costs in Experimental Constant Sum Games." Games and Economic Behavior, April 1997, 19(1), 97-132.

[27] Nelson, Richard R. and Winter Sidney G. An Evolutionary Theory of Economic Change. Harvard University Press, 1982.

[28] Nowak, Martin A.; Bonhoeffer, Sebastian and May, Robert M. "More Spatial Games." International Journal of Bifurcation and Chaos, February 1994, 4(1), $33-56$. 
[29] Nowak, Martin A. and May, Robert M. "Evolutionary Games and Spatial Chaos." Nature, October 29, 1992, 359, 826-9.

[30] ___-_. "The Spatial Dilemmas of Evolution." International Journal of Bifurcation and Chaos, February 1993, 3(1), 35-78.

[31] Radner, Roy. "Economic Survival." Nancy Schwartz Memorial Lecture, Northwestern University, 1996; reprinted in D. P. Jacobs, et al, Frontiers of Research in Economic Theory, Cambridge University Press, Cambridge, 1998, 183-209.

[32] Saez-Marti Maria and Weibull Jorgen W. "Clever Agents in Young's Evolutionary Bargaining Model." Journal of Economic Theory, June 1999, 86(2), 268-79.

[33] Salmon, Timothy. "An Evaluation of Econometric Models of Adaptive Learning." Econometrica, November 2001, 69(6), 1597-628.

[34] Samuelson, Larry. Evolutionary Games and Equilibrium Selection. Massachusetts, MIT Press, 1997.

[35] Stahl, Dale O. "Evolution of Smartn Players." Games and Economic Behavior, October 1993, 5(4), 604-17.

[36] Vega-Redondo, Fernando. "The Evolution of Walrasian Behavior." Econometrica, March 1997, 65(2), 375-84.

[37] Winter Sidney G. "Economic 'Natural Selection' and the Theory of the Firm." Yale Economic Essays, 1964, 4.

[38] ____-. "Satisficing, Selection, and The Innovating Remnant." Quarterly Journal of Economics, May 1971, 85(2), 237-61. 
[39] Young, H. Peyton. "The evolution of conventions." Econometrica, January 1993, $61(1), 57-84$.

[40] ___-_. Individual Strategy and Social Structure. Princeton University Press, 1998. 